\title{
Recognition of Corona virus disease (COVID-19) using deep learning network
}

\author{
Ashwan A. Abdulmunem, Zinah Abulridha Abutiheen, Hiba J. Aleqabie \\ Department of Computer Science, University of Kerbala, College of Science, Iraq
}

\begin{tabular}{l} 
Article Info \\
\hline Article history: \\
Received Apr 3, 2020 \\
Revised Jun 23, 2020 \\
Accepted Jul 10, 2020 \\
\hline Keywords: \\
Classification \\
Corona virus disease \\
COVID-19 \\
Deep learning \\
X-ray lung images
\end{tabular}

\section{Corresponding Author:}

Ashwan Anwer Abdulmunem,

Department of Computer Science, University of Kerbala,

Karbala, Iraq.

Email: Ashwan.a@uokerbala.edu.iq

\begin{abstract}
Corona virus disease (COVID-19) has an incredible influence in the last few months. It causes thousands of deaths in round the world. This make a rapid research movement to deal with this new virus. As a computer science, many technical researches have been done to tackle with it by using image processing algorithms. In this work, we introduce a method based on deep learning networks to classify COVID-19 based on x-ray images. Our results are encouraging to rely on to classify the infected people from the normal. We conduct our experiments on recent dataset, Kaggle dataset of COVID-19 X-ray images and using ResNet50 deep learning network with 5 and 10 folds cross validation. The experiments results show that 5 folds gives effective results than 10 folds with accuracy rate $97.28 \%$.
\end{abstract}

This is an open access article under the $\underline{C C B Y-S A}$ license.

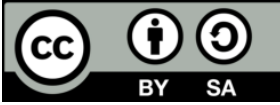

\section{INTRODUCTION}

A new virus disease spreads last December in Wuhan city in China for uncertain reasons and it was named by the World Health Organization (WHO) as COVID-19 [1]. Because this virus disease is new so there is no medicine is incapable of treating the symptoms whose associated with this virus disease for those people infected by. As a result, thousands of people around the world were passed away and for those who were recovered have different respiratory problems. This virus disease accessed the whole world in a state of panic and fear, and all works outside the home has been stopped as a result of the outbreak of this virus [2].

The incubation period of the virus disease is 1-14 days, at which time its symptoms appear fever, fatigue and dry cough in addition to shortness of breath, in this period the infected people are the source of infection, but it is possible to limit the spread of the virus and prevent infection through the early diagnosis of the disease or the virus carrier and the injured person is isolated and treat the patient [1]. One of the primary treatments is being worked out is recording X-ray for the patients to make sure of the patient's injury, where a lung part is taken to diagnose the condition of patient by the doctor.

After the revolution in technology is exploded, technology has an effective role in solving and tackle with most of life areas. One of these areas, medical field, where the computer algorithms was used to detect and classify different diseases by using machine learning techniques to analyze X-ray images and give desired results. X-ray considered as a digital image. Digitally, the image is represented by a number of pixels, and each pixel contains a specific value according to the nature of the image (RGB, gray, binary). There are a lot of applications can be implemented to analyze images. One of these applications, segmentation which depends on edges of object and extract common features [2]. There are many researches depend on 
segmentation in them researches to reach into specific result such as $[3,4]$ other researchers worked on review such as [2,5], also, segmentation challenges have been had in [6]. Main contributions of the work are:

- Working with a vital challenge which is Corona virus (COVID-19) recognition though limited available data, make this problem hard to deal.

- Use recent machine learning algorithms (deep learning) to process and recognize the hottest subject in the world. This work can help the health sector to speedup the digannoss the diseas at its earliest stages.

- Gain significat results to be the work more acceptable and reliable to prove the computerized alogorithms can help the community in the epidemic disasters.

The paper organized as following: section 2 reviews the related work and previous researches. Section 3 describes the proposed method: dataset and deep learning networks. While section 4 illustrates the results of the method on COVID-19 dataset. Finally, the conclusion and future work are drawn in section 5 .

\section{RELATED WORK}

COVID-19 classification has been an attractive area for researcher to develop algorithms dealing with this new challenge. Digital image processing technologies have been extensively used in the medical sector and prove the effectiveness of these algorithms with acceptable results. Therefore, these algorithms have been one of the hottest solutions to tackle with this problem. Because the urgent need to implement a reliable system to diagnose this virus disease. Recently, many works are introduced to classify and detect the disease in its earliest stages to save the patients' lives. As known, image processing algorithms have many applications on the health sector, including organ segmentation, diseases detection and classification, predication and more, offering support for eventual medical diagnosis [7].

A novel approach was suggested for X-ray segmentation [8] that detects lungs and ribs in chest radiographs. It lies on the fact that instead of using pixel-wise techniques exclusively the region based features were used as wavelet features that take into consideration the orientation of anatomic structures. Then classification applied of non-rib lung regions for radiographic patterns suggesting tuberculosis infection.

An algorithm has been developed [9] to diagnose chest X-ray pneumonia at a point that is exceeded by clinicians. It is the 121-layer neural network CheXNet, which has been trained on Chest X-rays 14 . The optimization of such a deep network was through dense connections and batch normalization. In addition, a multiple disease identification extension exceeding previous state of the art on Chest X-ray14 has also been presented.

The recent algorithms of image processing are deep learning networks and have an effective role to deal with this issue. The first attempt of using deep learning models established in [7] where Pulmonary CT images were taken to build a network of classifications for distinguishing between COVID-19 and viral pneumonia Influenza-A. The classic ResNet was used for feature extraction in terms of the network structure. The form of infection and a total confidence score for this CT case was determined using Noisy-or Bayesian function.

A deep-learning framework for segment and quantitative study of infection areas and of all lung from thorough CT scans of COVID-19 patients have been developed [10]. The system also does the auto-construct infectious areas but its contours, volumes and infection percentage (POIs) are precisely estimated in CT scans. A neural network "VB-Net" is utilized by the researchers on CT scans for segment COVID-19 infection regions. In training phase, 249 patients used with COVID-19 and validated with 300 new COVID-19 patients. While deep feature were suggested in [11] to train the support vector machine classifies the corona affected X-ray images from others. The transfer learning approach is applied for the identification of rice diseases in the before mentioned deep CNN models. The deep feature extraction based on deep learning architectures such as AlexNet, VGG16, VGG19, GoogleNet, ResNet18, ResNet50, ResNet101, InceptionV3, InceptionResNetV2, DenseNet201 and XceptionNet. The deep features obtained from these deep models are classified by SVM.

Nowadays, COVID-19 virus disease occupies the first attention in medical images processing field. Moreover, Deep Learning Networks prove that they are achieve the highest rates in different application (segmentation, classification and recognition) comparing to the standard algorithms. Based on these, the researchers proposed many algorithms to face the COVID-19 recognition challenge [7]. Relevant experiments were directed on the diagnosis of pulmonary nodules, X-rays image classification [12], pneumonia detection [9, 13], classification of benign tumors [14], and lung infection quantification [10]. Most articles cited in this section have used the images of ieee8023 [15] and Kaggle [16] as well as images from other public databases for other situations like COVID-19. 
Ozturk et al. [17] suggested a deep model of the use of X-ray images for early COVID-19 detection. In binary and multiclass modes, the author has performed the classification. For binary classes, the model is built with an accuracy of $98.08 \%$ and for the multi class case $87.02 \%$. The DarkNet model was constructed as a YOLO classification system for object detection. The authors have provided the codes and stated to use these tools for the validation of their primary screenings by radiologists.

Hemdan [18] suggested the use of COVIDX-Net in X-ray images to diagnose COVID-19. They have an accuracy of $90 \%$ using 25 positive and 25 normal images from COVID-19. Khan et al. [19] CoroNet has been designed to identify COVID-19 CXR images as a convolution neural network (CNN). For COVID-19 identification, The CoroNet's adjusted average accuracy is achieved by 0.87 and F1 by 0.93 . In [20] SqueezeNet has been shown to use Bayesian optimization to diagnose COVID-19 with an accuracy of 98.26 percent including its light network architecture.

Aimed at COVID-19 pneumonia screening [21], Farooq and Hafeez propose a 3-step development has been suggested to simplify the ResNet-50 pre-trained architecture to boost model efficiency and the training time, naming it COVID-ResNet. Multiclass Classification with a 96.23 percent accuracy model achieved.

In [22], a neural network that is a concatenation of the Xception and ResNet50V2 has been trained to classify X-ray images into normal, pneumonia, and COVID-19 classes. This network achieved for detecting COVID-19 cases is $99.50 \%$, and the overall average accuracy for all classes is $91.4 \%$. In [23], proposed a deep learning neural network-based method nCOVnet that considered a fast screening method to detect the COVID-19 by analyzing the X-rays of patients. The experimental result for 318 COVID-19 patients with $97.97 \%$, and 320 COVID-19 negative patients with $98.68 \%$. The proposed model correctly distinguishes positive COVID-19 patients for $97 \%$ precision, although its overall accuracy is $88 \%$.

In [24] a classification scheme was established in COVID-19 by introducing a multiclass classification, including a hierarchical criterion for pneumonia classification. Resampling algorithms have been used because of the natural data imbalance to re-balance the class distribution. Features were extracted utilizing a few well-known texture descriptors and a pre-trained model. For the COVID-19 detection, $0.65 \mathrm{~F} 1$-score were shown for multiclass and $0.89 \mathrm{~F} 1$-score.

Recognized COVID-19 on the use of artificial intelligence technologies in chest CT images in [25]. Addressed identification COVID-19 for chest CT images using the techniques of artificial intelligence. The dataset of six Chinese hospitals was made up of 2969 CT images taken from healthy individuals and other people suffering from COVID-19 and other lung diseases.

In [26], Develop AI-based CT image analysis software for identifying, quantifying, and monitoring coronavirus and demonstrate that they can differentiate patients with coronavirus from those who are not affected. Many international datasets were used. The studying resulted in 0.996 AUROC images of coronavirus compared to non-coronavirus images.

Deep CNN, equipped with [27] as COVID-Net to detect COVID-19 cases in chest X-ray (CXR) images, and the predictions made by COVID-Net were tested using the explainability approach to obtain more data, but also to validate COVIDENet's decision-making with accountability and clarity. Introduce also COVIDx, an open-access benchmark dataset, which was created with 13.975 CXR images in 13,870 patient cases, with the largest publicly available number of positive COVID-19. The accuracy of this model obtained $92.4 \%$.

In [28] the COVID-19 contaminated patients were identified using the deep transfer technique. In comparison, a top 2 smooth loss function with cost-sensitive characteristics has been dealt with the noisy and imbalanced datasets COVID-19. The results showed that up to $96.2264 \%$, and $93.0189 \%$ respectively were training and test accuracy. Depending on the success of the CNN, we use pre-trained deep neural network called ResNet50 to extract features and recognize the infected people by COVID-19 based on their $\mathrm{X}$-ray images. In the following sections, we will explain the details of the proposed method and the dataset we used in our work and discuss our results

\section{PROPOSED METHOD}

\subsection{Dataset}

We use the X-ray COVID-19 dataset from Kaggle website, this dataset collected by [29]. The dataset contains $50 \mathrm{X}$-ray images for lung, where $25 \mathrm{X}$-ray images for patients and $25 \mathrm{X}$-ray images for healthy patients. For experiments, the dataset divided into 40 observations (20 infected and 20 normal) for training and 10 (5 infected and 5 normal) observations for testing. Figure 1 explains some examples of the X-ray images in the Kaggale dataset that used in the experiments. 

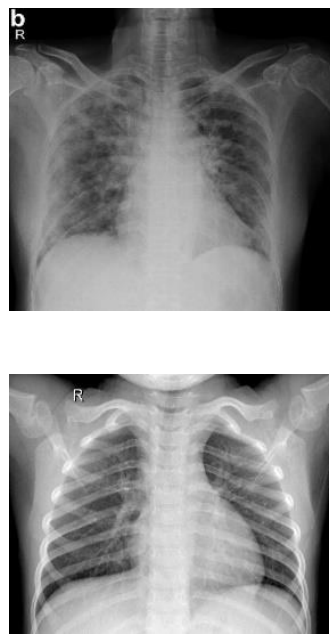

Normal

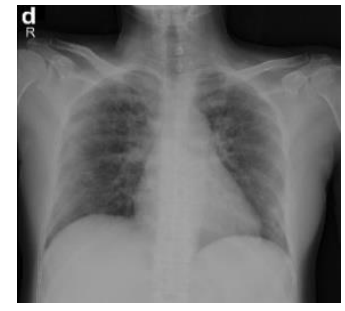

COVID

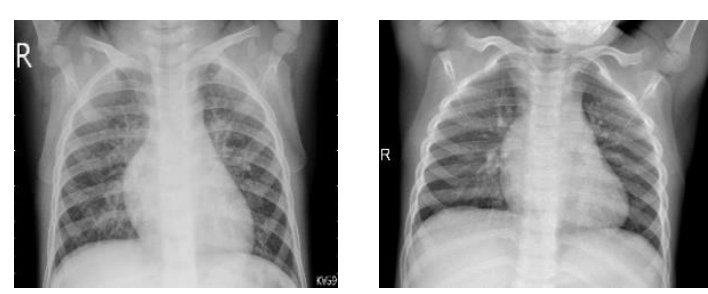

, First row for infected people with COVID-19,

Figure 1. Examples of X-ray images, First row for infected
Second row for normal people

\subsection{Deep learning network architecture}

In our implementation of the proposed method, we use ResNet50 deep learning network. The ResNet is a short name for residual network and 50 is the number of the layers in this network (deep). This network based on residual learning that means learning some residual instead of trying to learn other features. Residual can simply be interpreted as a subtraction of the function learned from that layer's data. ResNet does this by adding shortcut (directly adding nth layer input to any $(n+x)$ th layer). It has shown that training this type of networks is easier than training simple deep neural networks and solving the problem of degrading precision. The structure of the ResNet50 illustrated in Figure 2.

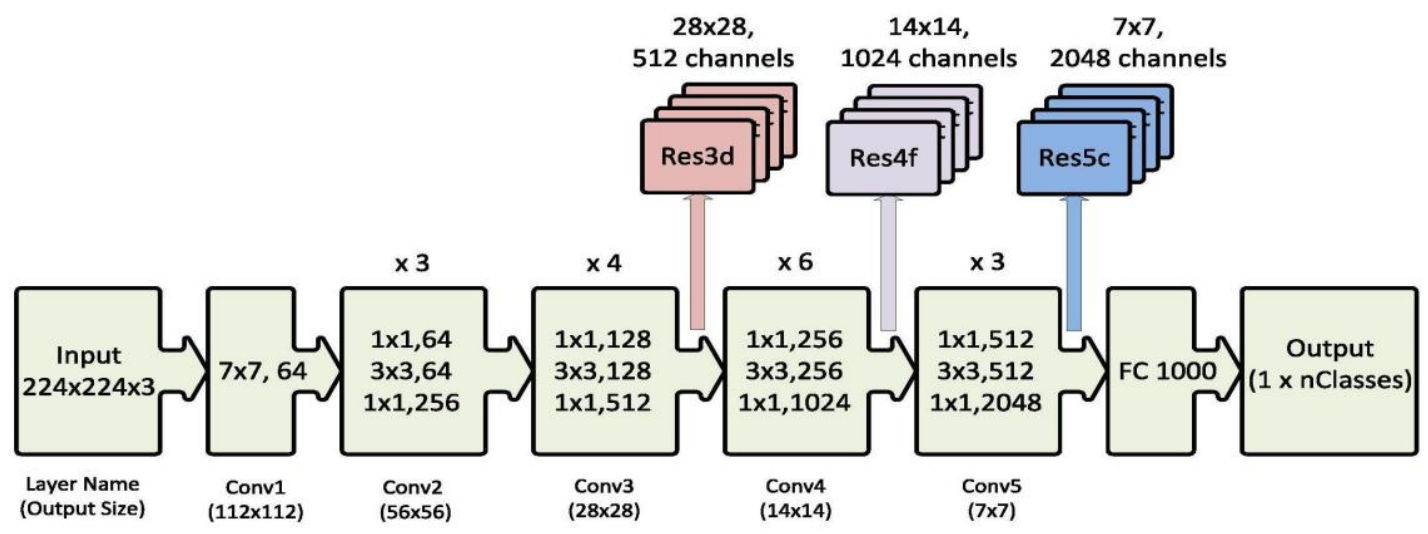

Figure 2. Structure of the ResNet50 deep learning network [30]

As a learning transfer in which the model trained on some task is reproduced to another task. This is usually done by adjusting toward the new task. In our case, COVID-19 detection works with small data, assuming the detection achieved on the model trained on ImageNet (which contains millions of labeled images. Therefore, transfer learning has a significant role to play in using this model with a limited dataset like COVID-19. Dropweights followed by a softmax activated layer are added to the network to estimate informative model uncertainty in a completely connected layer. The softmax layer outputs the probability distribution for all possible class labels. All images we redimensioned to 224 x 224 pixels. The images were standardized using mean and standard deviation values from the X-ray dataset. We divide the entire dataset between training and testing sets into 80\% -20\%. Data augmentation was also applied. All our experiments were performed for 30 epochs. 


\section{RESULTS AND DISCUSSION}

In this section, we will depict the results of the deep learning network (ResNet50). The basic idea of this network is utilizing the shortcut connections to bypass blocks of convolutional layers. "Bottleneck" blocks are the name of these basic blocks. These blocks obey two simple design rules:(i) the layers have the same number of filters for the same output feature size; and (ii) the number of filters is multiplied if the feature map size is halved. Therefore, the X-ray given image will pre-process by transforming it into grayscale image. After that, convert to 3-channel picture to match architecture of ResNet50 deep learning. And all images will be converted to $224 \times 224$ size to be accepted to manipulated into the input layer of the ResNet50 network. Then down-sampling is performed directly on convolutionary layers with a step of 2 and batch normalization is performed immediately after each revolution and before activation of rectified linear unit (ReLU).

According to the validation process, as mentioned in dataset section, the whole images in the dataset split into training set (40 images) and test set (10 images). These two sets include normal and covid $\mathrm{x}$-ray images. We use cross-validation to validate the outcomes. By choosing two values for $\mathrm{K}$ in two experiments $(\mathrm{k}=5$ and $\mathrm{k}=10)$. In first experiment $(\mathrm{k}=5)$, that means the complete collection of data is divided into $\mathrm{k}$-folds. A fold is selected one by one as test fold. Then, one by one, one of the remaining sets will be used as validation sets, and the other sets will be used as training sets until all possible combinations are tested. The accuracy and loss error of the Deep networks are shown in Figure 3 (see in Appendix) for 5-folds cross validation. The work is done by MATLAB (R2019a) with using Deep learning ToolBox. It can be observed in these figures that after 30 epochs both loss and accuracy continue to stabilize for all folds and the accuracy of both train and test sets is very high, converging to an accuracy of $(97.28 \%)$. While when using 10 folds the loss and accuracy charts will be as shown in Figure 4 (see in Appendix).

The accuracy reaches to $(95.99 \%)$. Moreover, we compute confusion matrix to show the true positive (TP), true negative (TN), false positive (FP) and false negative (FN) for both experiments (5-folds and 10-folds). Figure 5 shows the confusion matrices for 5-folds and 10-folds respectively. Table 1 illustrates the accuracy, precision, sensitivity parameters for testing set in each two experiments (5-folds and 10-folds). The parameters calculated based on the following equations:

\begin{tabular}{|c|c|c|c|c|c|}
\hline \multicolumn{3}{|c|}{ 5-folds } & \multicolumn{3}{|c|}{ 10-folds } \\
\hline & covid & normal & & covid & normal \\
\hline covid & 24 & 1 & covid & 23 & 2 \\
\hline normal & 1 & 24 & normal & 1 & 24 \\
\hline
\end{tabular}

Figure 5. Confusion matrices for 5-folds and 10-folds

$$
\begin{aligned}
& \text { Precision }=(\mathrm{TP}) /(\mathrm{TP}+\mathrm{FP}) \\
& \text { Sensitivity }=(\mathrm{TP}) /(\mathrm{TP}+\mathrm{FN}) \\
& \text { F-Measure }(\mathrm{F} 1)=2 \cdot((\text { Precision } \cdot \text { Sensitivity })) /(\text { Precision}+ \text { Sensitivity })
\end{aligned}
$$

Table 1. The results of the experiments

\begin{tabular}{lcccc}
\hline K-folds & Accuracy & Precision & Sensitivity & F-measure \\
\hline 5-folds & 0.9728 & 0.9600 & 0.9600 & 0.9600 \\
10-folds & 0.9599 & 0.9583 & 0.9200 & 0.9387 \\
\hline
\end{tabular}

From Table 1, it can be clearly seen that the recognition rate in 5-folds gives acceptable results than when train the dataset on 10-folds. The receiver operating characteristic (ROC) curve to demonstrate false positive rate and detection rate for 5-folds and 10-folds illustrate as shown in Figure 6. To compare our results with existence work, Table 2 illustrates this comparsion. 

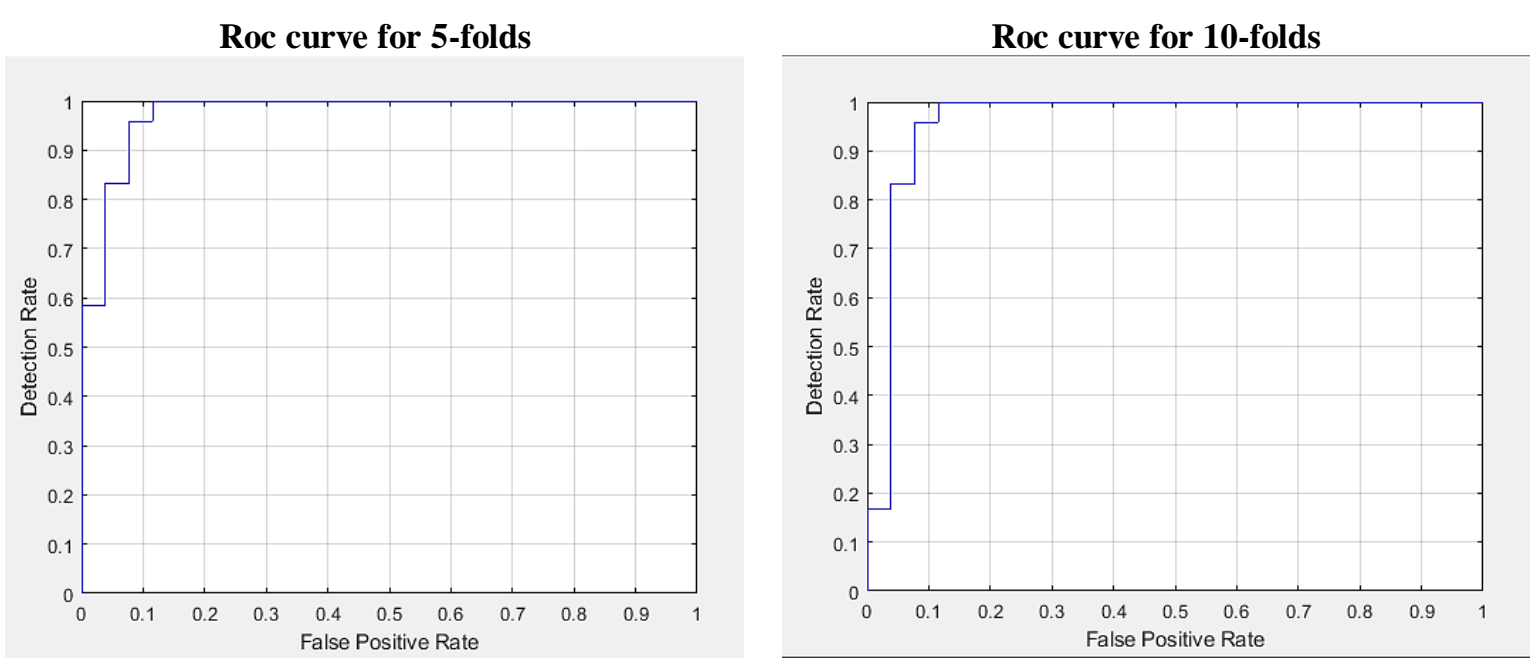

Figure 6. Roc curves for 5-folds and 10-folds cross-validation

Table 2. Deep learning techniques by using X-ray images for the prediction of COVID-19

\begin{tabular}{llll}
\hline Study & Deep learning Network & Dataset & Accuracy \% \\
\hline$[20]$ & Bayes-SqueezeNet-based & Ieee8023 +Kaggle & $\mathbf{9 8 . 2 6 \%}$ \\
{$[17]$} & DarkNet & Ieee8023 & $\mathbf{9 8 . 0 8 \%}$ \\
Proposed method & ResNet50 & Kaggle & $\mathbf{9 7 . 2 8 \%}$ \\
{$[21]$} & ResNet-50 & Preprocessed dataset of Ieee8023 and Kaggle & $\mathbf{9 6 . 2 3 \%}$ \\
{$[19]$} & CoroNet & Ieee8023+ Kaggle & $\mathbf{8 7 \%}$ \\
{$[28]$} & deep transfer learning technique & images are collected from various datasets & $\mathbf{9 3 . 0 1 8 9 \%}$ \\
{$[27]$} & COVID-Net & COVIDx dataset & $\mathbf{9 2 . 4 \%}$ \\
{$[22]$} & Xception and ResNet-50V2 & Ieee8023 +Kaggle & $\mathbf{9 1 . 4 \%}$ \\
{$[18]$} & COVIDX-Net & RYDLS-20 & $\mathbf{9 0 \%}$ \\
{$[24]$} & CNN & Ieee8023 & $\mathbf{8 9 \%}$ \\
{$[23]$} & nCOVnet & & $\mathbf{8 8 \%}$ \\
\hline
\end{tabular}

From Table 2, it is clear the proposed method performs significant results comparing with others. To sum up, the main contributions of this work are to deal with the vital problem the world face which is COVID19. The deep learning has significant results to recognize the virus in earliest stages so can save people lives. Moreover, the ResNet50 has a capability to deal with small dataset by using transfer learning by adopting the orignial model to deal with the newest task.

\section{CONCLUSION AND FUTURE WORK}

In this work we introduce a solution to solve the recent dilemma in the whole world. We use significant machine learning algorithms (Deep learning networks) which proves a notable success in medical community to deal with the tackle. The deep network that we use ResNet50.We evaluated the deep network on recent COVID-19 dataset from Kaggle website. The findings show that deep learning gives reliable results to classify the covid infected people based on their x-ray images with recognition rate is $97.28 \%$ in 5 -folds cross-validation experiments and $95.99 \%$ in 10 -folds cross validation experiments. As shown the recognition rate in 5-folds higher than the recognition in 10-folds. As a future work, we will conduct more experiments on different architecture of deep learning with different dataset to recognize the infected people in earlier stages and save their lives. In addition, we are planning to use Artificial intelligence algorithms to segment the covid infected parts in the lungs.

\section{ACKNOWLEDGEMENTS}

We are happy to thank our university (University of Kerbala) to support our work. 


\section{APPENDIX}

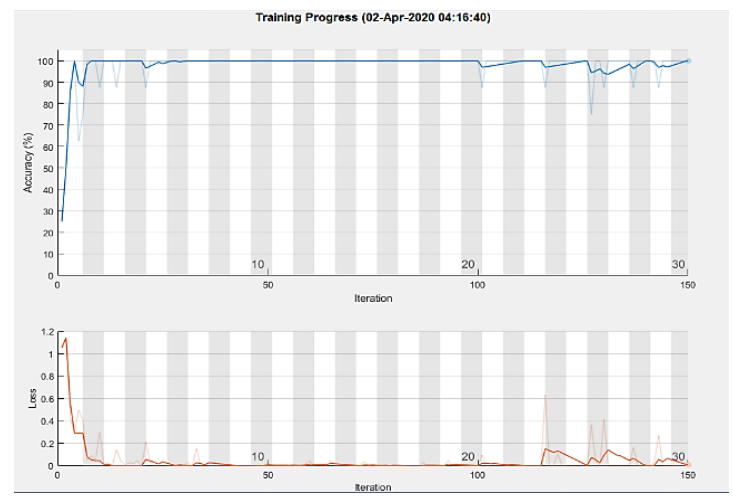

Fold 1

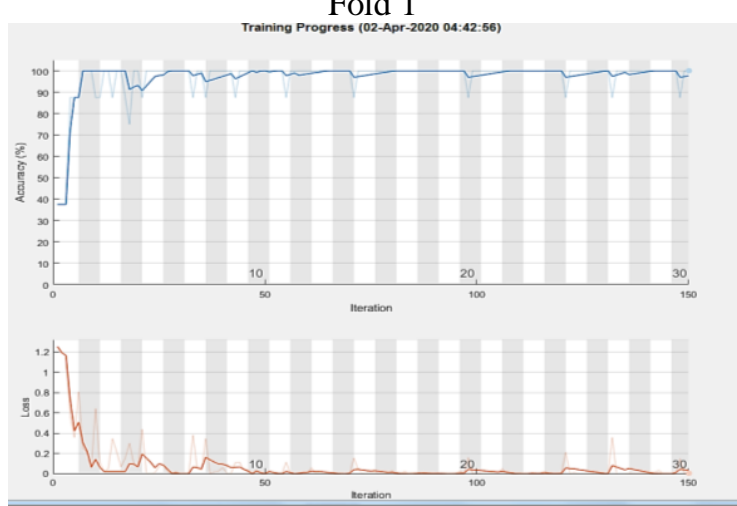

Fold 3
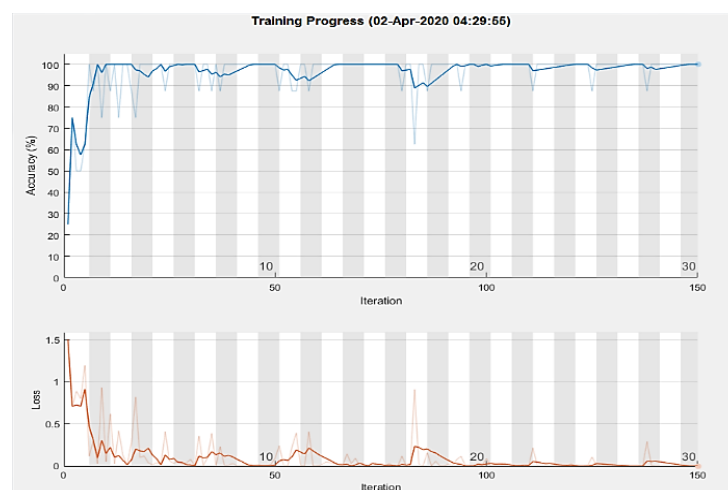

Fold 2

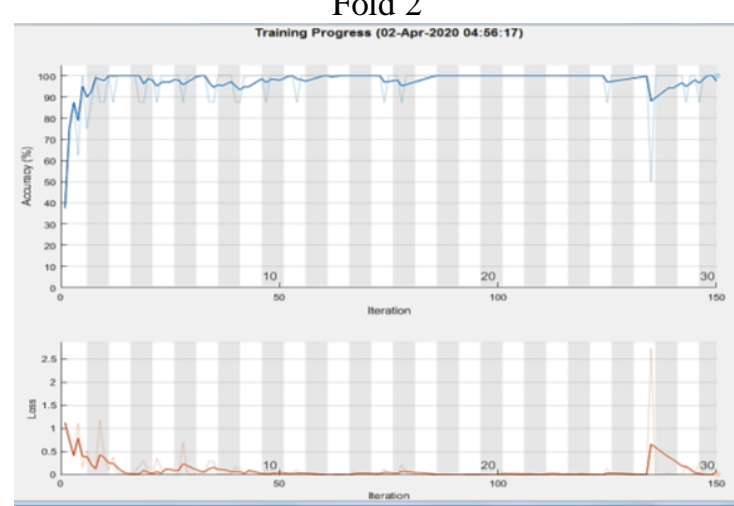

Fold 4
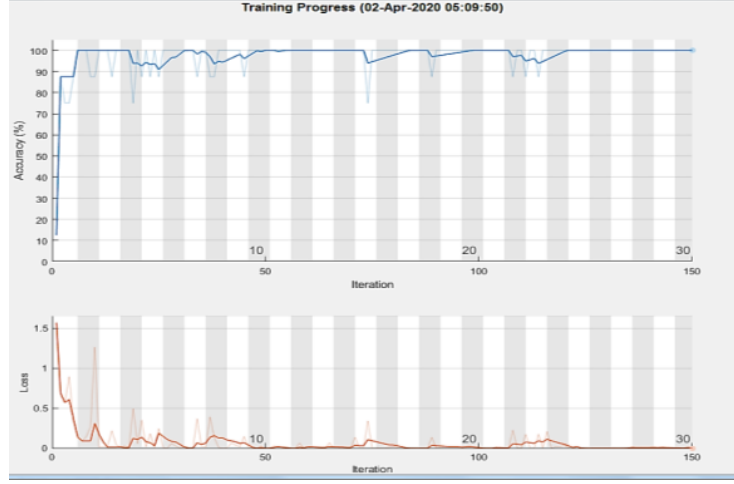

Fold 5

Figure 3. 5-folds training process for Kaggle dataset
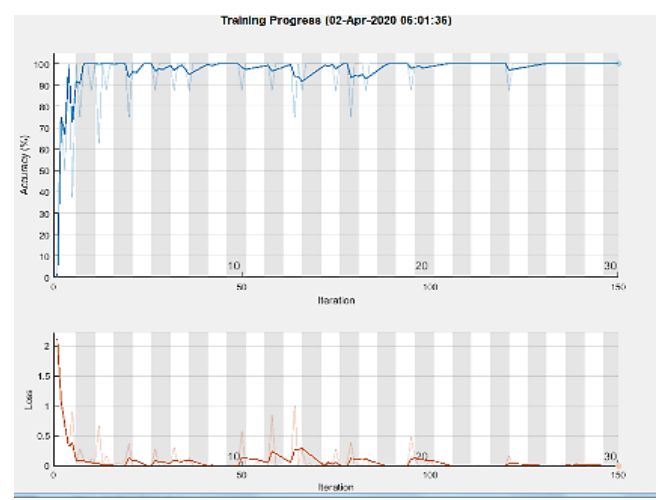

Fold1
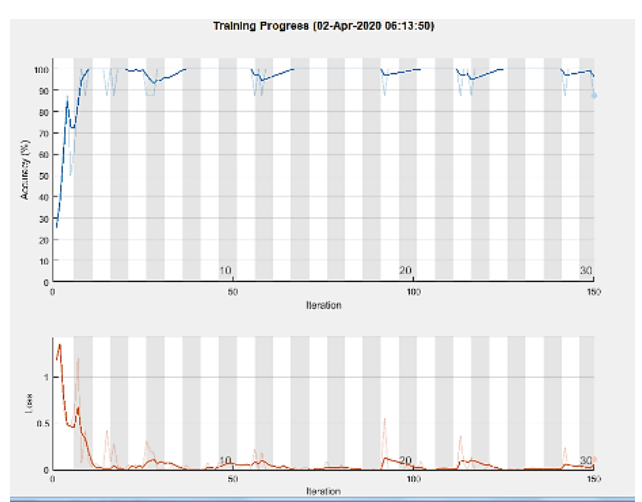

Fold2

Figure 4. 10-folds training process for Kaggle dataset 


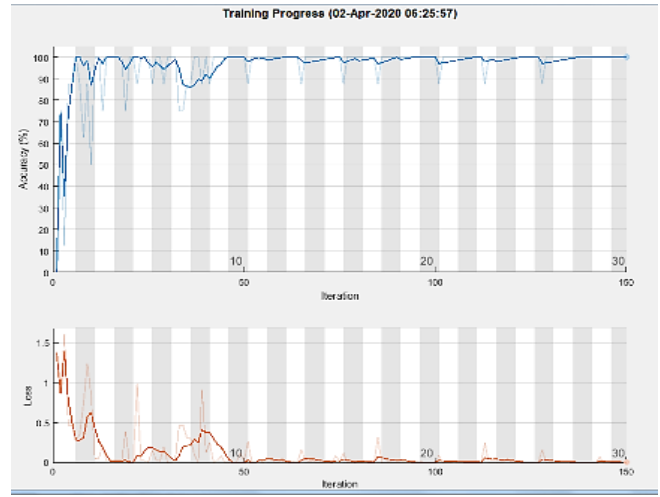

Fold3
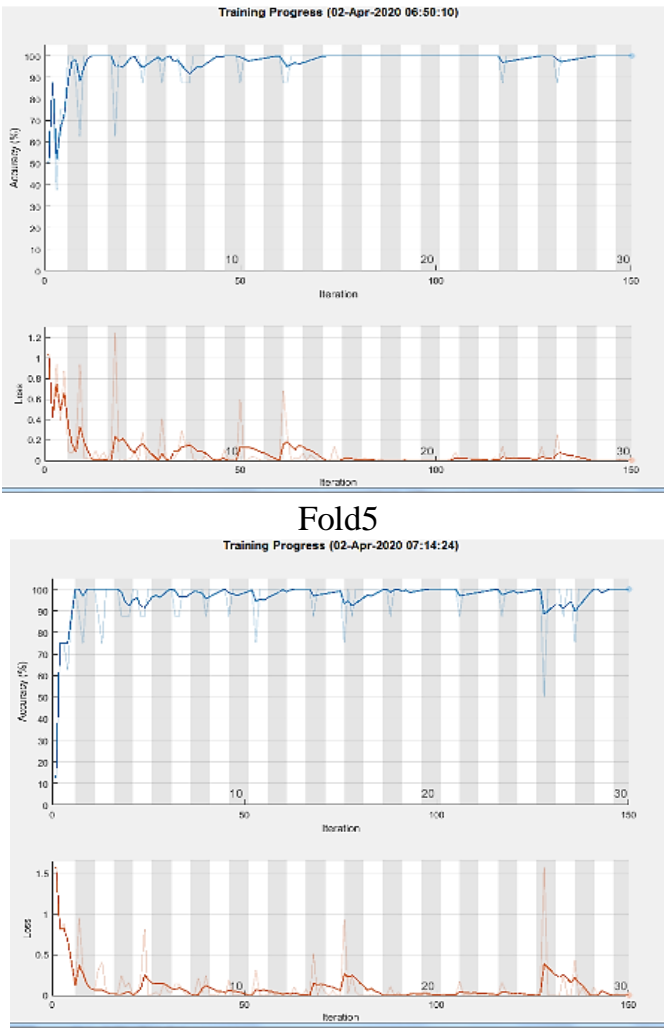

Fold7
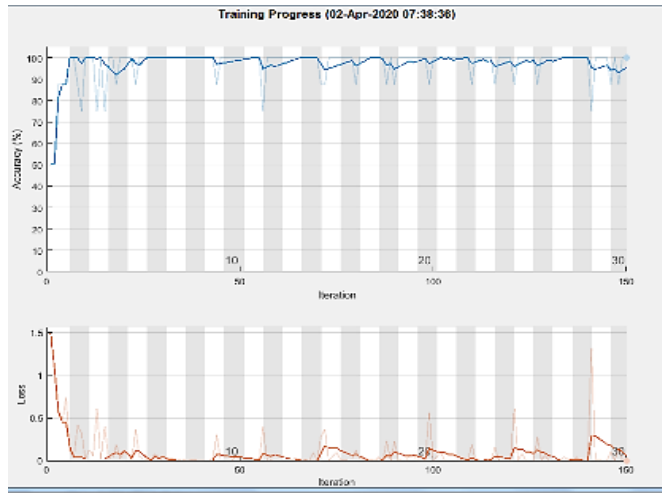

Fold9
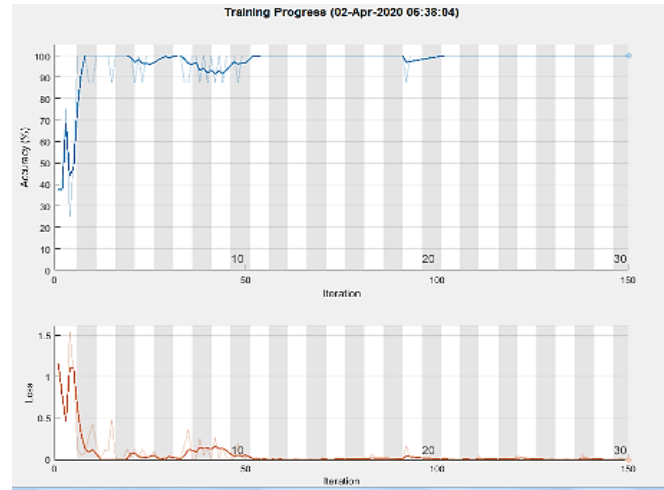

Fold4

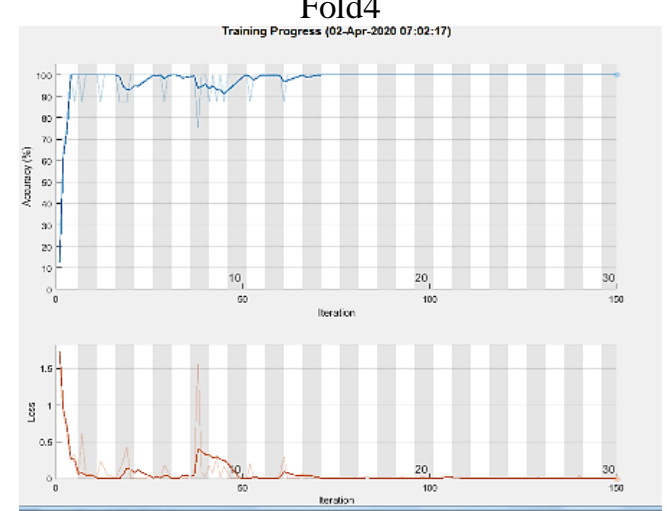

Fold6
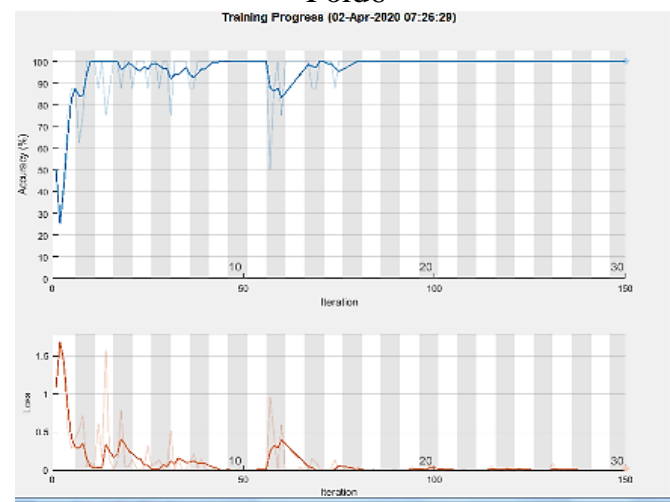

Fold8
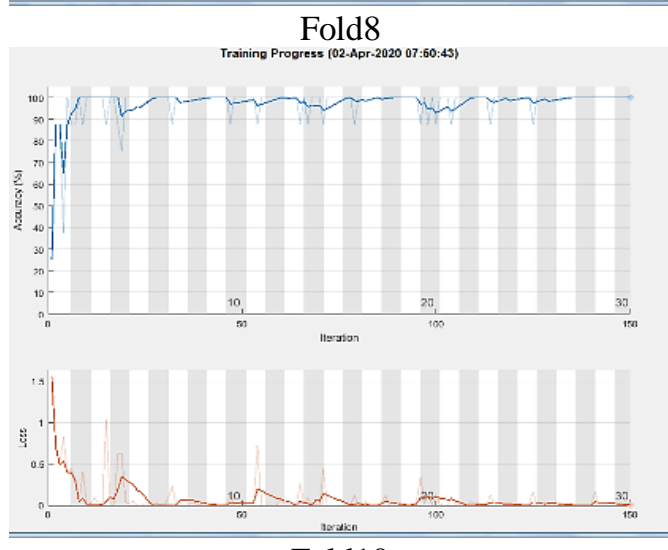

Fold10

Figure 4. 10-folds training process for Kaggle dataset (continue) 


\section{REFERENCES}

[1] Y. H. Xu et al., "Clinical and computed tomographic imaging features of novel coronavirus pneumonia caused by SARS-CoV-2," Journal of Infection., vol. 80, no. 4, pp. 394-400, 2020.

[2] N. R. Pal, and S. K. Pal, "A review on image segmentation techniques," Pattern recognition, vol. 26, no. 9, pp. 1277-1294, 1993

[3] E. Itje Sela, S. Hartati, A. Harjoko, and R. Wardoyo, "Segmentation on the Dental Periapical X-Ray Images for Osteoporosis Screening," Int. Journal of Advanced Computer Science and Applications, vol. 4, no. 7, 2013.

[4] S. Candemir et al., "Lung segmentation in chest radiographs using anatomical atlases with nonrigid registration," IEEE transactions on medical imaging, vol. 33, no. 2, pp. 577-590, 2014.

[5] G. Jader, L. Oliveira, and M. Pithon, "Automatic segmenting teeth in X-ray images: Trends, a novel data set, benchmarking and future perspectives," Expert Systems with Applications, vol. 107, pp. 15-31, 2018.

[6] V. Gayathri and H. P. Menon, "Challenges in Edge Extraction of Dental X-Ray Images Using Image Processing Algorithms-A Review," Citeseer, 2014.

[7] X. Xu et al., "Deep Learning System to Screen Coronavirus Disease 2019 Pneumonia," Applied Intelligence, pp. 1-29, 2020.

[8] A. Karargyris, S. Antani, and G. Thoma, "Segmenting anatomy in chest x-rays for tuberculosis screening," Annual International Conference of the IEEE Engineering in Medicine and Biology Society, pp. 7779-7782, 2011

[9] P. Rajpurkar et al., "CheXNet: Radiologist-Level Pneumonia Detection on Chest X-Rays with Deep Learning," arXiv preprint arXiv:1711.05225. pp. 3-9, 2017.

[10] Leontyeva S.V., Evstigneeva E.M., Lonina N.N., Kartsev G.N., Flid V.R., “Универсальный Синтез Замещенных Бицикло[2. 2. 1]Гептенов И Продуктов Их Аллилирования-Universal Synthesis Of Substituted Bicyclos [2.2.1] Heptenes And Their Allylation Products," Science Magazine, Basic research, no. 3, pp. 46-46, 2005.

[11] P. Kumar and S. Kumari, "Detection of coronavirus Disease ( COVID-19) based on Deep Features," Preprints, 2020030300, 2020.

[12] T. Rahmat, A. Ismail, and S. Aliman, "Chest X-rays Image Classification in Medical Image Analysis," Applied Medical Informatics, vol. 40, no. 3-4, pp. 63-73, 2018.

[13] V. Chouhan et al., "A novel transfer learning based approach for pneumonia detection in chest X-ray images," Applied Sciences, vol. 10, no. 2, 2020.

[14] A. Rezvantalab, H. Safigholi, and S. Karimijeshni, "Dermatologist Level dermoscopy skin cancer classification using different deep learning convolutional neural networks algorithms," arXiv preprint arXiv:1810.10348, 2018.

[15] J. P. Cohen, P. Morrison, and L. Dao, "COVID-19 image data collection," arXiv preprint arXiv:2006.11988, 2020.

[16] Paul Mooney, "Chest X-Ray Images (Pneumonia)," Kaggle. [Online]. Available: https://www.kaggle.com/paultimothymooney/chest-xray-pneumonia

[17] T. Ozturk, et al., "Automated detection of COVID-19 cases using deep neural networks with X-ray images," Computers in Biology and Medicine, 2020.

[18] E. E.-D. Hemdan, M. A. Shouman, and M. E. Karar, "COVIDX-Net: A Framework of Deep Learning Classifiers to Diagnose COVID-19 in X-Ray Images," arXiv preprint arXiv:2003.11055, 2020.

[19] A. I. Khan, J. L. Shah, and M. Bhat, "CoroNet: A Deep Neural Network for Detection and Diagnosis of Covid-19 from Chest X-ray Images," Computer Methods and Programs in Biomedicine, 2020.

[20] F. Ucar and D. Korkmaz, "COVIDiagnosis-Net: Deep Bayes-SqueezeNet based diagnosis of the coronavirus disease 2019 (COVID-19) from X-ray images," Medical Hypotheses, 2020.

[21] M. Farooq and A. Hafeez, "COVID-ResNet: A Deep Learning Framework for Screening of COVID-19 from Radiographs," arXiv preprint arXiv:2003.14395, 2020.

[22] M. Rahimzadeh and A. Attar, "A modified deep convolutional neural network for detecting COVID-19 and pneumonia from chest X-ray images based on the concatenation of Xception and ResNet50V2," Informatics Med. Unlocked, 2020.

[23] H. Panwar and R. Morales-menendez, "Application of Deep Learning for Fast Detection of COVID-19 in X-Rays using nCOVnet," Chaos, Solitons \& Fractals, 2020.

[24] R. M. Pereira, D. Bertolini, L. O. Teixeira, C. N. Silla, and Y. M. G. Costa, "COVID-19 identification in chest X-ray images on flat and hierarchical classification scenarios," Comput. Methods Programs Biomed., 2020.

[25] L Li, et al., "Artificial Intelligence Distinguishes COVID-19 from Community Acquired Pneumonia on Chest CT Lin," Radiology, 2020.

[26] O. Gozes, M. Frid, H. Greenspan, and D. Patrick, "Title: Rapid AI Development Cycle for the Coronavirus (COVID-19) Pandemic: Initial Results for Automated Detection \& Patient Monitoring using Deep Learning CT Image Analysis Article Type : Authors : Summary Statement : Key Results : List of abbreviati," arXiv preprint arXiv:2003.05037, 2020.

[27] L. Wang and A. Wong, "COVID-Net: A Tailored Deep Convolutional Neural Network Design for Detection of COVID-19 Cases from Chest X-Ray Images,” arXiv preprint arXiv:2003.09871, 2020.

[28] Y. Pathak, P. K. Shukla, A. Tiwari, S. Stalin, S. Singh, and P. K. Shukla, "Graphical abstract Deep Transfer Learning based Classification Model for COVID-19 Disease," IRBM, vol. 1, pp. 1-6, 2020.

[29] Adrian Rosebrock, "Detecting COVID-19 in X-ray images with Keras, TensorFlow, and Deep Learning," [Online] Available : https://www.pyimagesearch.com/2020/03/16/detecting-covid-19-in-x-rayimages-with-keras-tensorflowand-deep-learning, 2020.

[30] A. Singh and D. R. Kisku, "Detection of Rare Genetic Diseases using Facial 2D Images with Transfer Learning," 8th International Symposium on Embedded Computing and System Design, pp. 26-30, 2018. 


\section{BIOGRAPHIES OF AUTHORS}

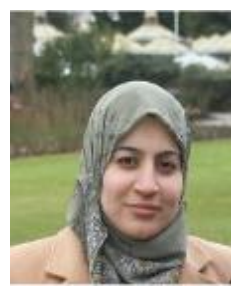

Ashwan Anwer Abdulmunem, received her Ph.D. degree in computer vision/Artificial Intelligence from Cardiff University, UK. Recentely, she is a lecturer at University of Kerbala. Her research interests include computer vision and graphics, pattern recognition, artificial intelligence, and deep learning networks.

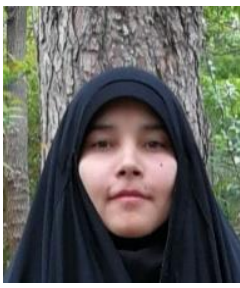

Zinah Abdulridha Abutiheen, Work at faculty of science Idepartment of computer science in university of Kerbala as a professor academy I 'm interesting NLP and image processing and computer vision researches.

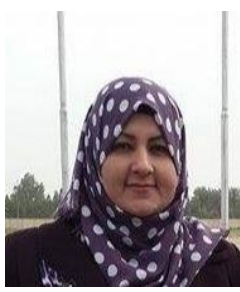

Hiba J. Aleqabie, Faculty member of Computer science, College of Science, Kerbala University, Karbala, Iraq. I did get my Ph.D. From The University of Babylon.M.sc.and B.Sc.from Al-Nahrain University. My research interest in multimedia processing and data mining.as well as twitter and socila networks mining. 